\title{
Recognition of a Face in a Mixed Document
}

\author{
Lhoussaine Bouhou ${ }^{1}$, Rachid El Ayachi², Mohamed Fakir ${ }^{3}$, Mohamed Oukessou ${ }^{4}$ \\ ${ }^{1,2,3}$ Computer Sciences Departement, Faculty of Science and Technology, \\ Sultan Moulay Slimane University, Morocco \\ ${ }^{4}$ Mathematics Departement, Faculty of Science and Technology, \\ Sultan Moulay Slimane University, Morocco \\ *Corresponding author, email: Ihoussbouh@yahoo.fr ${ }^{1}$, rachid.elayachi@usms.ma², fakfad@yahoo.fr ${ }^{3}$, \\ ouk_mohamed@yahoo.fr ${ }^{4}$
}

\begin{abstract}
Face recognition is the field of great interest in the domaine of research for several applications such as biometry identification, surveillance, and human-machine interaction. This paper exposes a system of face recognition. This system exploits an image document text embedding a color human face image. Initially, the system, in its phase of extraction, exploitis the horizontal and vertical histogram of the document, detects the image which contains the human face. The second task of the system consists of detecting the included face in other to determine, with the help of invariants moments, the characteristics of the face. The third and last task of the system is to determine, via the same invariants moments, the characteristics of each face stored in a database in order to compare them by means of a classification tool (Neural Networks and $K$ nearest neighbors) with the one determined in the second task for the purpose of taking the decision of identification in that database, of the most similar face to the one detected in the input image.
\end{abstract}

Keywords: histogram, humoments, Legendre moments, nearest neighbor, neural networks.

Copyright $(2015$ Institute of Advanced Engineering and Science. All rights reserved.

\section{Introduction}

The use of biometrics has two trends, the first is to facilitate the lifestyle and the second is to prevent fraud. However, face recognition is one of the biometric techniques. The difficulty of face recognition by computer depends on whether the conditions for acquisition. In a controlled environment, parameters such as the background, the direction and intensity of light sources, the angle of the shot, and distance from the camera to the subject are parameters controlled by the system. In an uncontrolled environment, a series of pretreatments are often required prior to the recognition itself. It must first detect the presence or absence of the face in the image. The face should then be segmented. Finally, if we are working on a video stream, the system must track the face from one image to the next. The problem of face recognition can be formulated as follows: Given one or more images of a face, the task is to find or verify a person's identity by comparing his face to the entire face images stored in a database. Additional information, such as race, age, gender, or speech can be used to reduce the search space. In general, a face recognition system usually consists of several steps to which our system, Nowadays Biometric systems are increasingly used. The use of the computer and its ability to process and store the data led to the creation of computerized biometric systems. There are several unique physical characteristics of an individual such as fingerprint face, voice recognition, DNA, etc. which explains the diversity of systems applying biometrics, as we take into account. Recently, the face recognition is attracting more and more attention. Security remains the primary area of application. In this area of the face recognition is responsible for the identification and authentication. Yang and Al have proposed a classification of methods of facial localization [1]:

a) "Knowledge-Based Methods": These methods to recognize different points of interest that make up a face and relationships between them. In Chiang et al., the problem in this type of method is that it is difficult to uniquely define a face [2].

b) "Feature invariant approaches": They are to use the elements invariant to changes in illumination, orientation or expression such as texture or the signature color of the skin for detection. 
c) "Template matching methods": Features models of a whole face or sub-part of the face (mouth, eyes, nose) are created. The location is then based on the correlation of these models with the candidates.

d) "Appearance-based methods": These are methods that use the same principle which was introduced in the previous paragraph except that they have the advantage to run very quickly but require a long period of training. The methods in this category have shown good results against 3 other methods include among them the method based on neural networks Rowley et al., the method of Schneiderman and Kanade based on a naive Bayes classifier and the famous Viola and Jones algorithm operating in real time [3-6].

The method of Viola \& Jones is a more efficient method at present. This allows to scan an image, using a detection window of initial size by $24 p x 24 p x$ and whether a face is present. When the image has been completely traversed, the window size is increased and the scanning is restarted, until the window size makes the image. Increasing the window size takes place by a multiplication factor of 1.25 .

As to scan it, it consists in shifting the window by one pixel. This shift can be changed to speed up the process, but a shift of one pixel ensures maximum accuracy which differentiates it from others [7]:

a) The use of integral images.

b) Selection by boosting features.

c) The combination boosted cascade of classifiers.

Selection by boosting is to replace a single classifier "strong" several "weak" classifiers in cascade. However, with a single classifier called "strong" should expect that the classifier is analyzed the entire window to determine whether a face is present in the image or not.

A cascade of classifiers whose selection criteria is less severe may replace the previous strong classifier. Consequently, as soon as one steps believes that there is no face, the window is rejected and the algorithm proceeds after which saves considerable time.

A methods based on neural networks can be summarized with the use of a classifier to two outputs representing the presence or absence of the object sought in a subregion of the image [8]. The basic principle is to scan the image with attention fixed dimensions and realize the detection of the sub-picture window.

There are other methods that are interested in the characteristic points of the face such as the nose, mouth and eyes [8]. The relative positions of different parts of the face are detected after being studied. However it there's difficulty encountered in this approach is to translate by strict rules to define how the researcher represents the face. If these rules are too specific, they do not recognize some faces. Otherwise, they cause false alarms. However, it allows a quick focus on the interesting parts of the image $[9,10]$.

The proposed approach in this paper is based on the use of two methods: The detection method for removing skin sections not having the color of the skin and that of the template matching for removing sections do not have the facial features [8].

This paper is organized as follows:

a) Section pixels representing skin from an input image.

b) Filtering skin areas.

c) Face detection by template matching "template matching".

Several studies have been based on the detection of skin. In most cases, skin is represented by a portion of a particular color space. Using the boundaries of this region as threshold values in an image, it is possible to extract the pixels whose color can be likened to that of the skin [11].

This is done in real time [12]:

a) The location of all the faces in the visual field,

b) Continued faces capture the visual field to another,

c) Facial recognition,

d) A backup of new faces in a database

e) Construction of the order of movement of the head to fix a particular person.

The method of face recognition is to convert images into local binary patterns, divided into several sub-regions to determine the identity of a face by comparing their histograms subregions.

The method of template or template matching, utilizes a comparison of the intensity of the pixels between a predefined template and several sub-regions of the image to be analyzed.

TELKOMNIKA Vol. 15, No. 2, August 2015 : $301-312$ 
This is in practice to perform multiple scans covering the entire area of the image. The most conducive to human faces areas will therefore easily identified by the minimum distance between the template and the underlying image [8].

The L1 norm (Manathan distance) and the L2 norm (Euclidean distance) of the standards are used in one distance possible in this method.

This method consists in performing the following steps:

a) Creation of the face model: The model will be a gray level image. Figure 1 shows examples of models [13]:

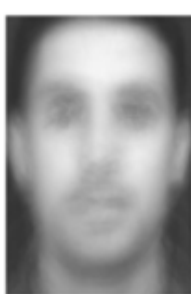

(a)

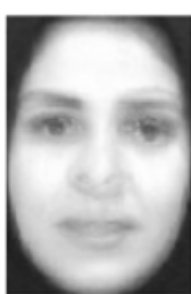

(b)

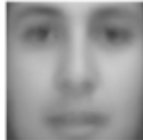

(c)

Figure 1. Examples of models of faces, (c) template used by this method [13]

b) Search for faces in skin regions: "Template Matching" since any image and a predefined model, determining the degree of membership (similarity) of this model to the image [14].

c) Similarity measures: There are several ways to measure the similarity between two images. The first measure applied by this method is a simple difference of standard data by the first formula for the L1 norm, and the second formula for the L2 norm.

Norm L1: $\quad \sum_{(i, j)}\left|a_{(i, j)}-b_{(i, j)}\right|$

Norm L2: $\left(\sum_{(i, j)}\left(a_{(i, j)}^{2}-b_{(i, j)}^{2}\right)^{2}\right)^{\frac{1}{2}}$

d) Application of the method of the "Template Matching" which requires the passage by the following steps:

1) Extraction of the image gray level,

2) Normalization of the segment,

3) Comparison of the segment with the model,

4) Decision.

A method of locating and extracting facial features using both shape and color [15] was proposed by Sobottka and Pitas [16]. This method consists in the segmentation of the image in the HSV color space to locate the region of the skin. And by applying a region growing algorithm with a coarse resolution, the connected components are then determined. For each connected component, the algorithm adjusts an ellipse in order to determine the candidate area which corresponds to the face. Finally, a more detailed analysis of features within this region leads to the conclusion about the presence of a face or not [17].

In $[18,19]$ a method is used to classify the pixels of skin color based on a Gaussian model for the skin color. A set ofelevn lower-order geometrical moment is calculated using the Fourier transform and the radial Mellin transform to characterize the shape of "clusters" in the binarized image. To detect the face region, a neural network is trained using the extracts geometrical moments [17].

In this paper Figure 2 illustrated the recognition system adopted. 


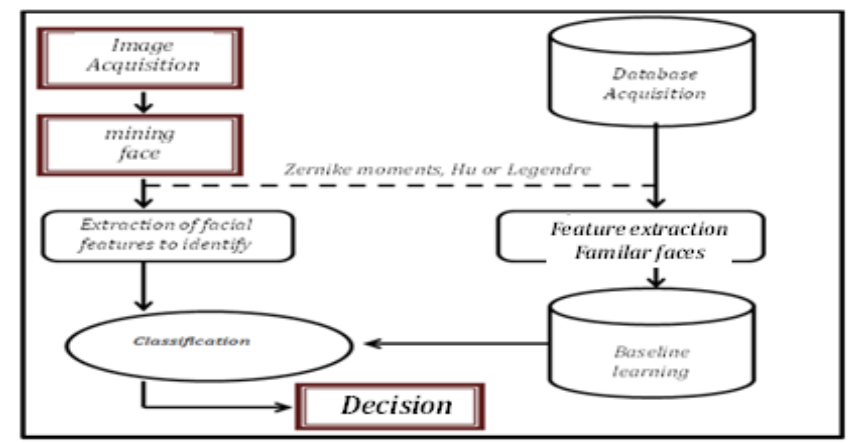

Figure 2. Face recognition system

\section{Extraction}

The extraction step is the key step in the process, because the performance of the whole system depends on it. In this step also known as indexing or modeling, is extracted from the face image information that can model the face of a person by a measurement vector that characterizes (feature vector or signature).

The judicious choice of the extraction method is extremely important insofar as the next step (classification) will be made only on the basis of the values of these vectors. Extraction methods are numerous, among them, Zernike moments, Hu and Legendre are mentioned.

\subsection{Zernike Moments}

Zernike moments are often used to capture the global features of an image recognition and image analysis. Introduced for the first time in computer vision by Teague [20], this shape descriptor has proven its superiority over other functions moments in its ability description and robustness to noise and distortion. Very recently, many researchers have looked at these moments, mainly to optimize computation time and improve accuracy.

We denote by Zernike moments a series of calculations used to transform an image into a vector of real components representative moments $\mathrm{Zij}$ [21].

The main advantage that this Zernike moments are insensitive to translation, rotation and scaling which preserves the information contained in the images [21].

Mathematically, Zernike moments are defined with a per order and repetition q on:

$$
\begin{aligned}
& \mathrm{D}=\{(p, q)|0 \leq p \leq \infty,| q|\leq p,| p-q \mid=\text { pair }\}: \\
& Z_{p q}=\frac{p+1}{\pi} \iint_{x^{2}+y^{2} \leq 1} \mid V_{p q}^{*}(x, y) f(x, y) d x d y
\end{aligned}
$$

Where $\mathrm{V}_{\mathrm{pq}}^{*}$ denotes the complex conjugate of $\mathrm{V}_{\mathrm{pq}}$ himself defined as:

$$
V_{p q}(\rho, \theta)=R_{p q}(\rho) \cdot e^{i q \theta}
$$

And,

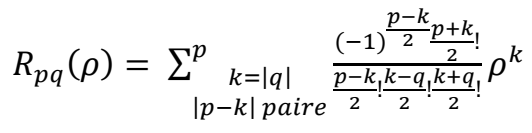

From the Equation (1) and (2), we can express Zernike moments of a rotated image of an angle $\alpha$ around its origin in polar coordinates:

$$
Z_{p q}^{\propto}=Z_{p q e^{i q \alpha}}
$$

The Equation (3) proves the invariance rotation of Zernike moments module since | $Z_{p q} e^{i q \alpha}|=.| Z_{p q} \mid$ Thanks to the orthogonality property, image reconstruction can be simply expressed as the sum of each basic function weighted by the corresponding Zernike moment: 


$$
\tilde{f}(x, y)=\sum_{(p, q) \in D} \sum Z_{p q} V_{p q}(x, y)
$$

\subsection{Hu Moments}

In [22], from the geometrical moments, Hu [23] proposed a set of seven moment invariants to translation, rotation and scaling. They are widely used in the literature for describing forms for a classification or indexing, but are quite sensitive to noise. Moreover, this family of descriptors is neither orthogonal nor complete.

The formula of the moment of Hu $m_{p q}$ order $p+q$ of a distribution function $f(x, y)$ is as follows:

$$
m_{p q}=\int_{-\infty}^{+\infty} \int_{-\infty}^{+\infty} x^{p} y^{q} f(x, y) d x d y
$$

For a digital image $g(x, y)$ of size $M * N$, the above formula becomes:

$$
m_{p q}=\sum_{y=0}^{N-1} \sum_{x=0}^{M-1} x^{p} y^{q} g(x, y)
$$

The central moment $\mu_{\mathrm{pq}}$ is given by:

$$
\mu_{p q=\sum_{y=0}^{N=1} \sum_{x=0}^{M-1}(x-\dot{x})^{p}(y-\dot{y})^{q} g(x, y)} \text { avec } \dot{x}=\frac{m_{10}}{m_{00}}, \dot{y}=\frac{m_{01}}{m_{00}}
$$

The normalized central moment is defined:

$$
\eta_{p q}=\frac{\mu_{p q}}{\mu_{00}^{\gamma}}, \gamma=\frac{p+q}{2}, p+q=2,3, \ldots
$$

\subsection{Legendre Moments}

Legendre moments have been introduced by Teague [24]. They have been used in many applications of pattern recognition [25].The times for a discrete Legendre image $\mathrm{M} \times \mathrm{N}$ pixels with intensity function $f(x, y)$ is [26]:

$$
\mathrm{L}_{\mathrm{pq}}=\lambda_{\mathrm{pq}} \sum_{\mathrm{x}=0}^{\mathrm{M}-1} \sum_{\mathrm{y}=0}^{\mathrm{N}-1} \mathrm{P}_{\mathrm{p}}\left(\mathrm{x}_{\mathrm{i}}\right) \mathrm{P}_{\mathrm{q}}\left(\mathrm{y}_{\mathrm{j}}\right) \mathrm{f}(\mathrm{x}, \mathrm{y})
$$

Where $\lambda_{p q}=\frac{(2 p+1)(2 q+1)}{M * N}$

$x_{i}$ and $y_{j}$ denote the normalized pixel coordinates in the range $[1,1]$, which are given by:

$$
\left\{\begin{array}{l}
x_{i}=\frac{2 x-(M-1)}{M-1} \\
y_{j}=\frac{2 y-(N-1)}{N-1}
\end{array}\right.
$$

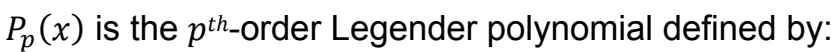

$$
P_{p}(x)=\sum_{k=0}^{p}\left\{\frac{(-1)^{\frac{p-k}{2}} x^{k}(p+k)}{2^{p} k !\left(\frac{p-k}{2}\right) !\left(\frac{p+k}{2}\right) !}\right\}_{p-k=\text { even }}
$$

And, the recurrent formula of the Legendre polynomials is:

$$
\left\{\begin{array}{c}
P_{p}(x)=\frac{(2 p-1) x}{p} P_{p-1}(x)-\frac{p-1}{p} P_{p-2}(x) \\
P_{1}(x)=x, P_{0}(x)=1
\end{array}\right.
$$

In which work the recurrent formula is used for calculating Legendre polynomials in order to increase the computation speed. 


\section{Classification}

The classification is the assignment of a specific class or not a given face class: class here is a person with face images in the database and the non-class in the case where the face belongs any class. This allocation requires the introduction of a measure of similarity.

\subsection{K-Nearest Neighbor}

K-Nearest Neighbor (K-NN) is part of supervised learning which has been used in many applications including data mining, statistical pattern recognition and image processing.

The algorithm does not construct a classification model but it is based on values found in the storage or memory. To identify the class of an input, the algorithm selects the class to which the majority of the $\mathrm{k}$ nearest neighbors belong entry.

The K-nn algorithm is considered one of the simplest machine learning algorithms. However, it is computationally expensive especially when the size of the training set becomes large which would cause the classification task to be very slow.

The following Figure 3 illustrates the modeling of this algorithm.

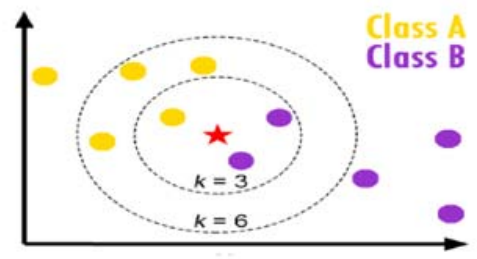

Figure 3. K-NN

\subsection{Neural Networks}

It is a set of interconnected neurons for formal resolution of complex problems such as pattern recognition or natural language processing, by adjusting weights in a learning phase.

The operation of the neural network based on the functioning of biological neurons and is embodied in a form of a computer algorithm. Neural networks is known for its strength in the field of face recognition approach.

\subsubsection{Biological Neurons}

In very simplistic, a neuron is a biological cell that is characterized by synapses, dendrites, axons and core.

The biological living cells are neurons which are interconnected by links called axons [27] leading the electric signals from the output of a neuron to the input (synapse) of another neuron.

\subsubsection{Artificial Neuron}

A neural network consists of simple elements, operating in parallel, which were inspired by the biological nervous system. It consists of a weighted directed graph whose nodes symbolize the formal neurons. These have an activation function to influence other neurons in the network. Connections between neurons, the so-called synaptic links, propagate the activity of neurons with characteristic weighting of the connection.

It may cause a neural network for a specific job (for example, character recognition) by adjusting the values of the connections (or weight) between the elements (neurons).

The formal neuron is an elementary unit of a neural network. It performs the weighted sum of its inputs, as the value of this summation is compared with a threshold. The output of the neuron is:

$$
\begin{aligned}
& \mu=\sum_{i=1}^{n} w_{i} \cdot e_{i}-\theta \\
& \mathrm{Y}=f(\mu)
\end{aligned}
$$


Where,

$e_{i}$ : element of the input vector,

$\mathrm{i}=1 \ldots \mathrm{n}$ : entries formal neuron,

$\mathrm{y}$ : output,

$w_{i}$ : weighting parameters,

f: activation function.

\section{Results and Analysis}

The objective of this section is to present the recognition system (whose steps are shown in Figure 4 adopted for the recognition of a face in a text document, and the results found in each phase of the system.

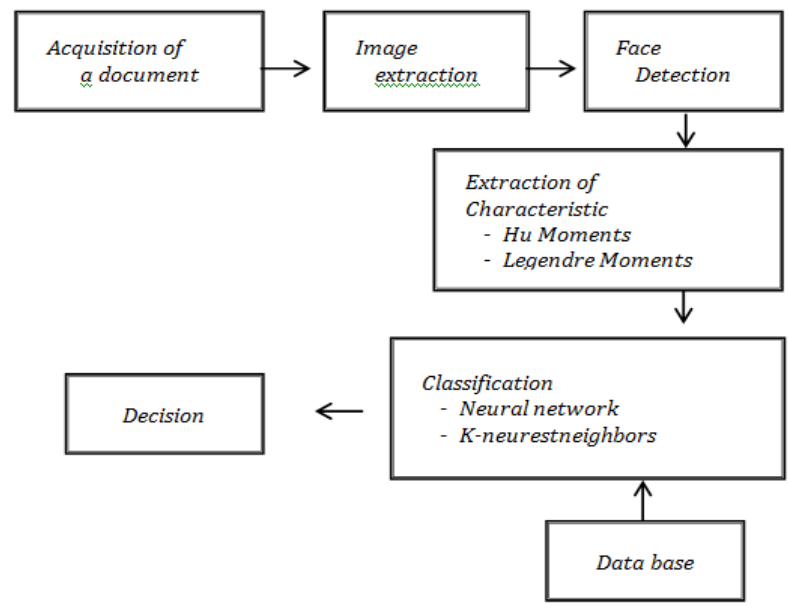

Figure 4. Recognition system

The documents presented in Figure 5 show two examples treated by our system using an interface developed in MATLAB, which offers different menu to choose, for each test, both the descriptor to be used in extraction level and the classifier to use in the classification level.

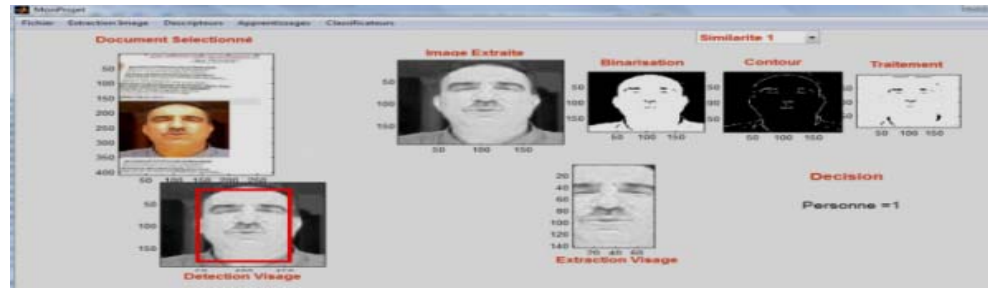

(a)

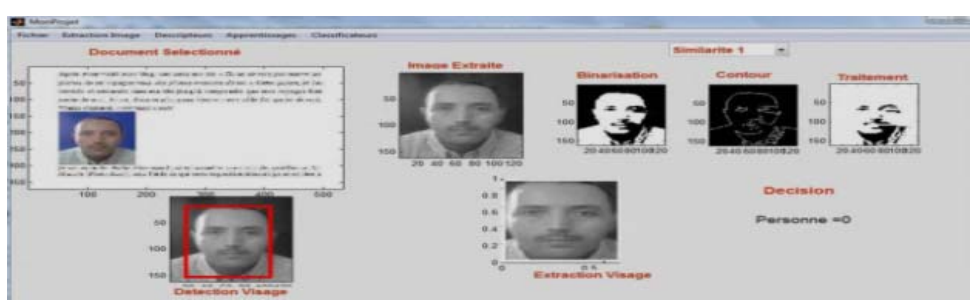

(b)

Figure 5. Examples of documents processed by our system 


\subsection{Data Base}

There was a database of 20 faces (Figure 6) spread over 5 different classes of people with 4 face varied, depending on the facial expressions of the person and the distance characterizing the image of the same person at the time from his grasp.

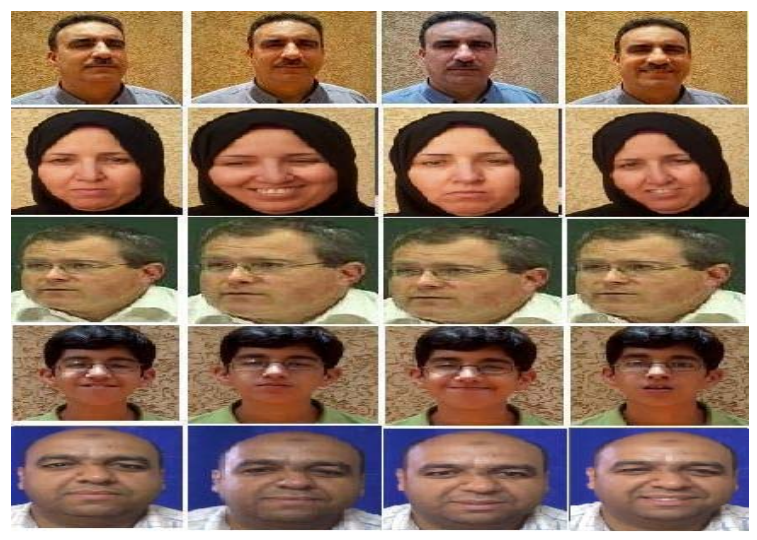

Figure 6. Database used by the developed system

This database is transformed, during the treatment, two reference bases, one for the $\mathrm{Hu}$ Moments descriptor, and the other by the Legendre moments descriptor.

\subsection{Face Recognition System}

To describe this system, we present here different phases.

Phase 1: Selection of a document

Detecting a face in a document is as easy without hypothesis. This is why our system will consider the hypothesis of a text document containing a single image (without the presence, in the picture, other figures that can disrupt system performance). So we collected different document glad both text and an image containing a face to select (Figure 7) one of these documents will be the main element of which came from the system must extract the face portion to be subsequently compared with those in the database to identify the person in the input image.

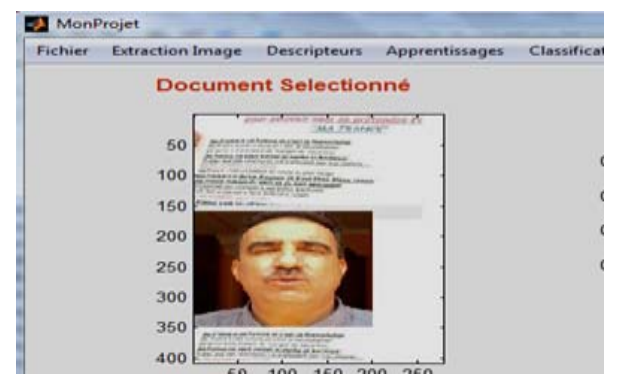

Figure 7. Example of selecting a document

Phase 2: Image extraction

This phase was carried out in two steps:

a) Step 1: The vertical histogram of the document has allowed us to determine the first and last references $x_{1}$ and $x_{2}$ pixel (vertically) of the image in the document, as illustrated in Table 1, to determine the extraction of the first original document was indeed able to extract, of the document, by these two part unlimited points $x_{1}$ and $x_{2}$. 
b) Step 2: based on the portion of material extracted in step 1, the horizontal histogram has allowed us to determine the first and last reference pixel $x_{3}$ and $x_{4}$ (horizontally) in the image of the document as it is shown in Table 1 to determine the extraction of the second extraction previous indeed it has been extracted, of the document, by these two part unlimited point $x_{3}$ and $x_{4}$.

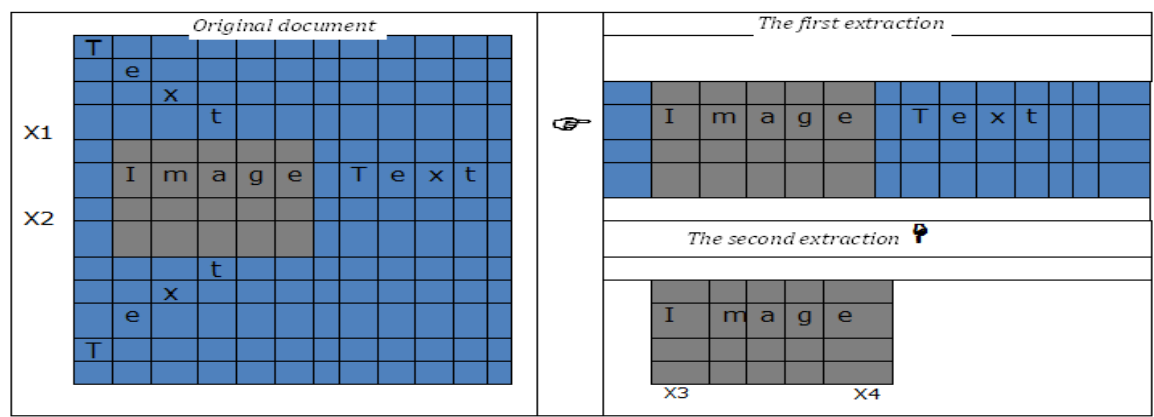

Table 1. Steps of extracting the image of the document

This phase allows us, therefore, as shown in Figure 8, to define the image area containing the face, from the initial document for use in the following phases.

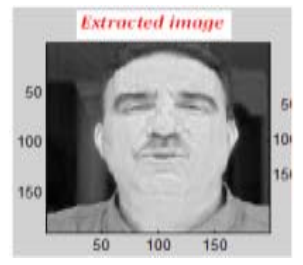

Figure 8. Extraction of the document image

Phase 3: Face detection image

This phase starts with a pretreatment on the extracted image in the previous phase containing the face detecting to determine the contour of the face followed by a processing for locating the face portion of the non-face part (Figure 9) to extract only the face on which the system will build in the next phase.

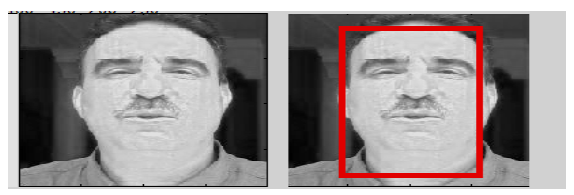

Figure 9. Face Detection

Phase 4: Extraction of facial features

Was used in this phase, the time and the moment of Hu Legendre (detailed in Chapter 2) to obtain, on the one hand, the facial features detected in the previous phase, and create, Moreover, for each moment, a reference database, for all the images of the database. Each of these basewill be operate in the next phase depending on the choice of classifier used in this phase. 
Phase 5: Classification

Neural network, first requires an initial training step which will be defined in the construction of the multilayer structure of the neural network (the number of inputs of the network depends on the size of the feature vector, the number cells of the hidden layer and the number of desired output), for our system, these inputs 7 indicates characteristics of $\mathrm{Hu}$ moments, the cells 10 and hidden layer 20 corresponding to the number of outputs in the base face data Figure 10. Learning ends when the algorithm reaches the stop criterion defined by our system in 1000 iterations.

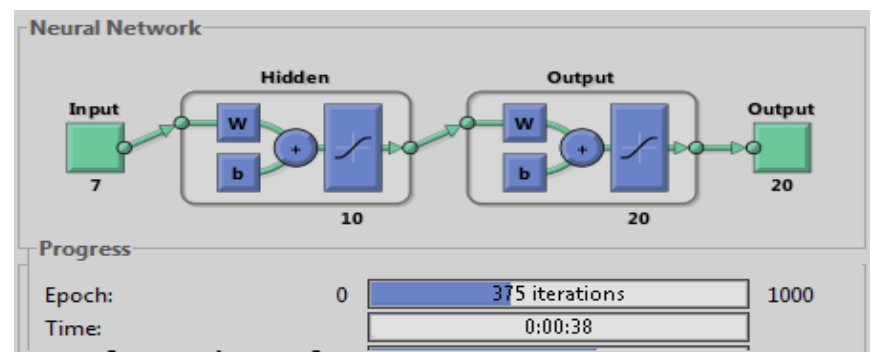

Figure 10. Phase learning neural net

Once the network has acceptable performance, it can be used for recognition of a possible phase in a second step, this phase of testing, by Hu Moments of the facial features on the image those of the output of the network to make a decision on the result of the classification.

We also used the descriptor Legendre Moments to order 3, which allows us to have 10 features for each face of the database (and the structure of the neural network, in this case, is 10 entries indicating characteristics of Legendre moments, 10 cells hidden layer and 20 outputs corresponding to the number of faces in the database) that will be tested with those of the detected face to be there too, a decision on the classification results.

Experimental results obtained by the use of $\mathrm{Hu}$ moments and moments of the Legendre at extraction phase attribute, and neural networks and the K-nearest neighbors (k-nn) at the classification phase are illustrated in Table 2 shows the recognition rate (RR) and Error Rate (ER) calculated for different approaches to a database of 20 faces.

Table 2. Experimental Results

\begin{tabular}{lllllll}
\hline \multirow{2}{*}{ Descriptor } & \multicolumn{2}{l}{ Neural } & \multicolumn{4}{c}{ K-NN } \\
& \multicolumn{2}{l}{ Networks } & \multicolumn{2}{l}{ Euclidean } & \multicolumn{2}{c}{ Manhattan } \\
& R.R & E.R & R.R & E.R & R.R & E.R \\
\hline Hu Moments & $70 \%$ & $30 \%$ & $65 \%$ & $35 \%$ & $60 \%$ & $40 \%$ \\
Legendre Moments & $72 \%$ & $28 \%$ & $68 \%$ & $32 \%$ & $65 \%$ & $35 \%$ \\
\hline
\end{tabular}

Several similarities have been proposed during the experiments for the classification by the K-nearest neighbors and only similarities with the Euclidean distance and the Manhattan distance were selected for the continuation of our project.

We did some tests on the images we have taken a personal photo-pairs and other downloaded from the Net. The tests are performed on faces that come in different colors and different orientations and scales. We note in:

a) Figure 5(a) positive recognition because, for each of these three cases, the decision is correct because each person to be identified is in the database.

b) Figure 5(b), gain positive recognition because in this case the decision is also correct that the person to be identified is not in the database. 


\section{Conclusion}

We proposed in this paper a recognition system faces. This system basically revolves around two parties. The first part is the extraction of an image, present in a text document, from which, face detection will be established with the aim to determine the characteristics of the faces. And a second part which in the classification occurs when the extraction characteristics of each person's face stored in a database to determine the person whose face is like the face of the picture in the text document.

In the first part, we used the vertical histogram and horizontal histogram to determine which area of the paper tests, presents a high level of gray that matches the picture in the document. Treatment with determining the outline of this picture brought us to frame the face lying in the image to retrieve and then extract its features.

The second part uses the advantages of neural networks and K-Plus close neighbor who reside in their ability to machine learning, their problem solving without the need to write complex rules and reduce computational cost in phase classification. Among the perspectives of this work, we propose to exploit and develop the method used by the system to take into account other assumptions for both the type of document text and the image containing the face to extract the document. Finally, we must establish decision rules after the classification phase for face detection without using registered in the database, face different situations, the same person to minimize the execution time of a once the capacity of the data base is reduced while maximizing the rate of recognition.

\section{References}

[1] Ming-Hsuan Yang, David J Kriegman, Narendra Ahuja. Detecting faces in images: A survey. IEEE Transactions on pattern analysis and machine intelligence. 2002; 24(1): 1746-1762.

[2] Cheng-Chin Chiang, Wen-Kai Tai, Mau-Tsuen Yang, Yi-Ting Huang, Chi-Jaung Huang. A novel method for detecting lips, eyes and faces in real time. Real-Time Imaging. 2003; 9(4): 277-287.

[3] Wenlong Zheng, Suchendra M Bhandarkar. Face detection and tracking using a boosted adaptive particle Iter. Journal of Visual Communication and Image Representation. 2009; 20(1): 9-27.

[4] HA Rowley, S Baluja, T Kanade. Neural network-based face detection. IEEE Transactions on Pattern Analysis and Machine Intelligence. 1998; 20(1): 23-38.

[5] H Schneiderman, T Kanade. Probabilistic modeling of local appearance and spatial relationships for object recognition. Computer Vision and Pattern Recogni-tion, IEEE Computer Society Conference. 1998: 45

[6] Paul Viola, Michael Jones. Robust real-time object detection. In Second international workshop on statistical and computational theories of vision. Vancouver, Canada. 2001.

[7] Lobodol. Face tracking: implémentation de la méthode de Viola \& Jones. http://www.firediy.fr/article18.html. 2013.

[8] Ch Bencheriet, AH Boualleg, H Tebbikh, B Guerzize, W Belguidoum. Détection de Visages par Méthode Hybride. LAIG, Université. Guelma 24000, Algérie.

[9] A lemieux. Système d'identification de personnes par Vision numérique. 2003.

[10] Diane FERGUSON, François LEVASSEUR. Détection de visages. LAPI - ISICO. 2004.

[11] Garcia C, Tziritas G. Face Detection Using Quantized Skin Color Regions Merging and Wavelet Packet Analysis. IEEE Transactions on Multimedia. 1999: 264-277.

[12] Mathieu Van Wambeke. Reconnaissance et suivi de visages et implémentation en robotique tempsréel. Université Catholique de Louvain Ecole Polytechnique de Louvain. Mémoire de fin d'études. 2010.

[13] J Serra. Image analysis and mathematical morphology. Orlando, FL, USA: Academic Press, Inc. 1989.

[14] H Chang, U Robles. Face Detection. 2000.

[15] J Revaud, G Lavoué, A Baskurt. 'Une nouvelle mesure de distance entre descripteurs de moments de Zernike pour une similarité optimale une similarité optimale entre les images. CNRS, INSA-Lyon, LIRIS. France

[16] K Sobottka, I Pitas. Extraction of facial regions and features using color and shape information. In: Proc. 13th Internat. Conference Pattern Recognition. Vienna, Austria. 1996: 421-425.

[17] K Sobottka, I Pitas. A novel method for automatic face segmentation, facial feature extraction and tracking. Signal Processing Image Community. 1998; 12: 263-281.

[18] SOUHILA GUERFI ABABSA. 'Authentification d'individus par reconnaissance de caractéristiques biométriques liées aux visages 2D/3D. THĖSE, UNIVERSITE D'EVRY VAL D'ESSONNE. 2008.

[19] JC Terrillon, M David, S Akamatsu. Automatic Detection of Human Faces in Natural Scene Images by Use of a Skin Color Model and Invariant Moments. Proc. Third Int'l Conf. Automatic Face and Gesture Recognition. 1998: 112-117. 
[20] JC Terrillon, M David, S Akamatsu. Detection of Human Faces in Complex Scene Images by Use of a Skin Color Model and Invariant Fourier-Mellin Moments. Proc. Int'l Conference Pattern Recognition. 1998: $1350-1355$.

[21] J Revaud, G Lavoué, A Baskurt. Une nouvelle mesure de distance entre descripteurs de moments de Zernike pour une similarité optimale une similarité optimale entre les images. CNRS, INSA-Lyon, LIRIS. France. 2010.

[22] Leila Chergui, Maamar Kef, Mohammed Benmohammed. La Théorie de la Résonance Adaptative et les Moments de Zernike pour la Reconnaissance de Mots Arabes Manuscrit. Université Larbi Ben Mhidi, Département d'informatique, Oum El Bouaghi - Algérie. 2009.

[23] Alain Boucher, TA Anh Phuong. Caractérisation quantitative des bases d'images. TIPE, Institut de la Francophonie pour l'Informatique. Hanoi. 2006.

[24] MK Hu. Visual Pattern Recognition by moment invariants. IRE Transaction on Information Theory. 1962; 8(2): 179-187.

[25] MR Teague. Image analysis via the general theory of moments. J. Opt. Soc. Amer. 1980; 70: 920-930.

[26] Chee-Way Chonga, P Raveendranb, R Mukundan. Translation and scale invariants of Legendre moments. Pattern Recognition. 2004; 37: 119-129.

[27] M OUJAOURA, B MINAOUI, Mohammed FAKIR. Multilayer Neur al Networks and Nearest Neighbor Classifier Performances for Image Annotation. (IJACSA), International Journal of Advanced Computer Science and Applications. 2012; 3(11).

[28] R EL AYACHI. 'Reconnaissance des Formes par une Classe d'Invariants et Applications à la reconnaissance des documents Tifinaghes. THESE. 2012; 104: 48-56. 